\title{
A Design of a Signal Acquisition System for Underwater Physical Field
}

\author{
Jian Yang ${ }^{1, a}$ \\ ${ }^{1}$ The Chinese People’s Liberation Army Unit 91550, Dalian, 116023, China \\ aemail: yangjian_DL@163.com
}

Keywords: Underwater physical field; signal acquisition; system design

\begin{abstract}
In the test of underwater vehicle, obtaining all kinds of effective information of underwater vehicle in time and accurately is of great significance to the evaluation of test results. This paper presents a design of underwater physical field signal acquisition system, analyzes the requirements of the system, gives the overall framework, and introduces the implementation details of the system from the aspects of software structure and configuration files.
\end{abstract}

\section{Introduction}

In the test of underwater vehicle, the test organization needs to get all kinds of effective information of underwater vehicle in time and accurately[1], so that the test results can be effectively evaluated. In addition to the information of the motion and state of the target, the information of the environmental physical field of underwater test is very important, and it is an important basis for the evaluation of the test results[2]. This paper presents a design of underwater physical field signal acquisition system, analyzes the requirements of the system, gives the overall framework, and introduces the implementation details of the system from the aspects of software structure and configuration files.

\section{System requirement analysis}

The physical field signal acquisition system is an underwater data collector based on the Windows CE embedded operating system[3]. It uses the sensing device to obtain the acoustic, magnetic and hydraulic data related to the underwater target, and receives various operating instructions from the upper machine through a custom communication bus, such as the serial port, the USB bus or the Ethernet interface. The working state and task completion of the terminal are fed back to the host computer[4]. When the underwater detection mission is finished, the slave computer transfers the collected data stored in the electronic card to the host computer through the universal data bus interface.

The system needs to collect acoustic signals, magnetic signals and water pressure signals and save them[5]. If there is no water depth data, we should first collect the water depth signal and send the depth signal through the $\mathrm{D} / \mathrm{A}$ to the conditioning board. The acquisition of water depth signals is prior to the acquisition of acoustic magnetic signals[6].

There are two ways of working on the lower computer: online working mode and independent working mode. Work independently in accordance with the pre-set mode.

1) Continuous working mode: collecting continuously and repeatedly according to the specified voyage time.

2) Regular working mode: continuous collection according to the set time.

3) Watch work mode: collect according to the value of the signal, begin to collect when the signal is more valuable, and stop the acquisition when the signal is lost.

4) Joint working mode: collect data according to the set time and value.

Under the online working mode, the lower computer receives various operating instructions from the host computer through a custom communication bus (such as serial port, USB bus or Ethernet interface, and currently is a serial port), and feedback the working state and task completion of the slave computer to the host computer. 


\section{System software architecture}

The software system consists of two parts: the pre-set system and the acquisition system. The pre-set system runs independently, and is used to pre configure the working parameters needed for the acquisition system, and is saved in the configuration file system.ini. When the collection system starts running, it reads the configuration files and gets the corresponding working parameters, and works accordingly[7]. The relationship is shown in Figure 1. Using the factory software presupposition method can make all kinds of embedded intelligent devices work independently and do not need manual intervention. When the work mode or management configuration needs to be changed, only the instruction can be realized through the communication bus.

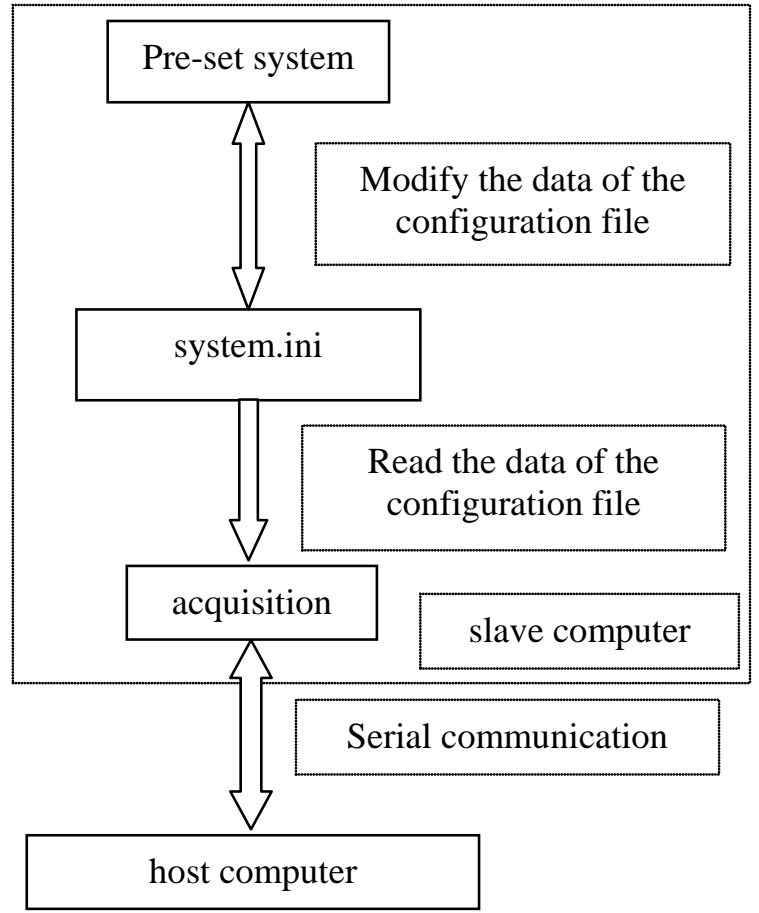

Fig.1. Relationship between subsystems

\section{System hardware architecture}

The hardware of the host and slave computer uses a high-performance, low power XScale processor, providing a bus extension interface such as PC/014, CF card, RS232, USB2.0 and Ethernet; use RS232 bus and USB bus to receive control instructions from the console and download the underwater data collection data in the CF card.

The system takes PC104 embedded industrial control computer as the core, 16 channel 16 bit A/D high speed band buffer data acquisition card, 4 A/D channels and 412 bit D/A channels for 24 bits complex precision magnetic field and water pressure field signal acquisition, large capacity array electronic disk, water depth measurement system, and digital I/O connection (partial operation and interruption), RS232 Interface, computer standard peripherals, and so on. These interfaces can easily interconnect with underwater acoustic communication interface, GPS time signal, parameter preset and data transcriptional equipment.

\section{Software implementation of the system}

The software of the slave computer consists of two parts, the data acquisition system and the pre-set system, which use the Windows CE 5 as the embedded operating system, and use the Embedded Visual C++ 4.2 as the development environment.

The project type of data acquisition system is Windows CE MFC Application, and the project 
name is Data Applicaion. The project type of the preset system is Windows CE MFC Application, and the project name is Preset. In addition to the files generated automatically by the development environment, the main source documents included in the system are shown in tables 1 and 2.

Tab.1. File List of Data Sampling Subsystem

\begin{tabular}{|c|c|c|}
\hline File name & class & describe \\
\hline CharQueue.h & \multirow{2}{*}{ CCharQueue } & \multirow{2}{*}{$\begin{array}{l}\text { Listen to the command buffer } \\
\text { queue, parse the command }\end{array}$} \\
\hline CharQueue.cpp & & \\
\hline MyComm.h & \multirow{2}{*}{ СМуСОММ } & \multirow{2}{*}{$\begin{array}{l}\text { Monitor the serial buffer, } \\
\text { identify the command }\end{array}$} \\
\hline MyComm.cpp & & \\
\hline PCM8308BS_Noise.h & \multirow{2}{*}{ CPCM8308BS_NOISE } & \multirow{2}{*}{ Acquisition of sound signals } \\
\hline PCM8308BS_Noise.cpp & & \\
\hline PCM8416AS_MagAndPress.h & \multirow{2}{*}{ CPCM8416AS_MAGANDPRESS } & \multirow{2}{*}{ Acquisition of pressure signal } \\
\hline PCM8416AS_MagAndPress.cpp & & \\
\hline PCM8308BS_ShuiShen.h & \multirow{2}{*}{ CPCM8308BS_SHUISHEN } & \multirow{2}{*}{$\begin{array}{l}\text { Acquisition of magnetic } \\
\text { signals }\end{array}$} \\
\hline PCM8308BS_ShuiShen.cpp & & \\
\hline MyStructDef.h & & Custom type \\
\hline $\begin{array}{l}\text { Profile.cpp } \\
\text { Profile.h }\end{array}$ & CProfile & Read and write INI file \\
\hline sysconfig.ini & & configuration file \\
\hline $\begin{array}{l}\text { ZwaPCM8308BSDrv.dll } \\
\text { ZwaPCM8308BSDrv.h } \\
\text { ZwaPCM8308BSDrv.lib }\end{array}$ & & PCM8308BS API \\
\hline $\begin{array}{l}\text { PCM8416AS_DRV.lib } \\
\text { pcm8416as_drvexport.h }\end{array}$ & & PCM8416AS API \\
\hline $\begin{array}{c}\text { epcIsa.h } \\
\text { epcIsa.lib } \\
\text { epcSerial.dll } \\
\text { epcSerial.h } \\
\text { epcSerial.lib } \\
\end{array}$ & & EPC8000 API \\
\hline $\begin{array}{l}\text { DataApplicationDlg.cpp } \\
\text { DataApplicationDlg.h }\end{array}$ & CDataApplicationDlg & $\begin{array}{l}\text { After the master control, the } \\
\text { command is parsed, the } \\
\text { custom notification message } \\
\text { dispatch function is executed. }\end{array}$ \\
\hline $\begin{array}{l}\text { PromptDelayDlg.cpp } \\
\text { PromptDelayDlg.h }\end{array}$ & CPromptDelayDlg & \\
\hline
\end{tabular}

Tab.2. File List of Presetting Subsystem

\begin{tabular}{|c|c|c|}
\hline File name & class & describe \\
\hline $\begin{array}{c}\text { Profile.cpp } \\
\text { Profile.h }\end{array}$ & CProfile & $\begin{array}{c}\text { Read and write INI } \\
\text { file }\end{array}$ \\
\hline sysconfig.ini & & configuration file \\
\hline $\begin{array}{c}\text { Preset.cpp } \\
\text { Preset.h }\end{array}$ & CPreset & $\begin{array}{c}\text { Execute the read and } \\
\text { write operation of the } \\
\text { configuration file }\end{array}$ \\
\hline
\end{tabular}

\section{Configuration system file}

For the convenience of the system, use the configuration file system.ini to save the working parameters. When the system starts running, it reads the configuration files, gets the corresponding working parameters, and works accordingly.

The pre-set system runs independently, and is used to pre configure the working parameters needed for the acquisition system.

The format of the configuration file system.ini is as follows:

[ACQUSITION]

bValidDepth $=0$

fDepth=10

;0-- No depth information,1- Deep information

; Deep information 


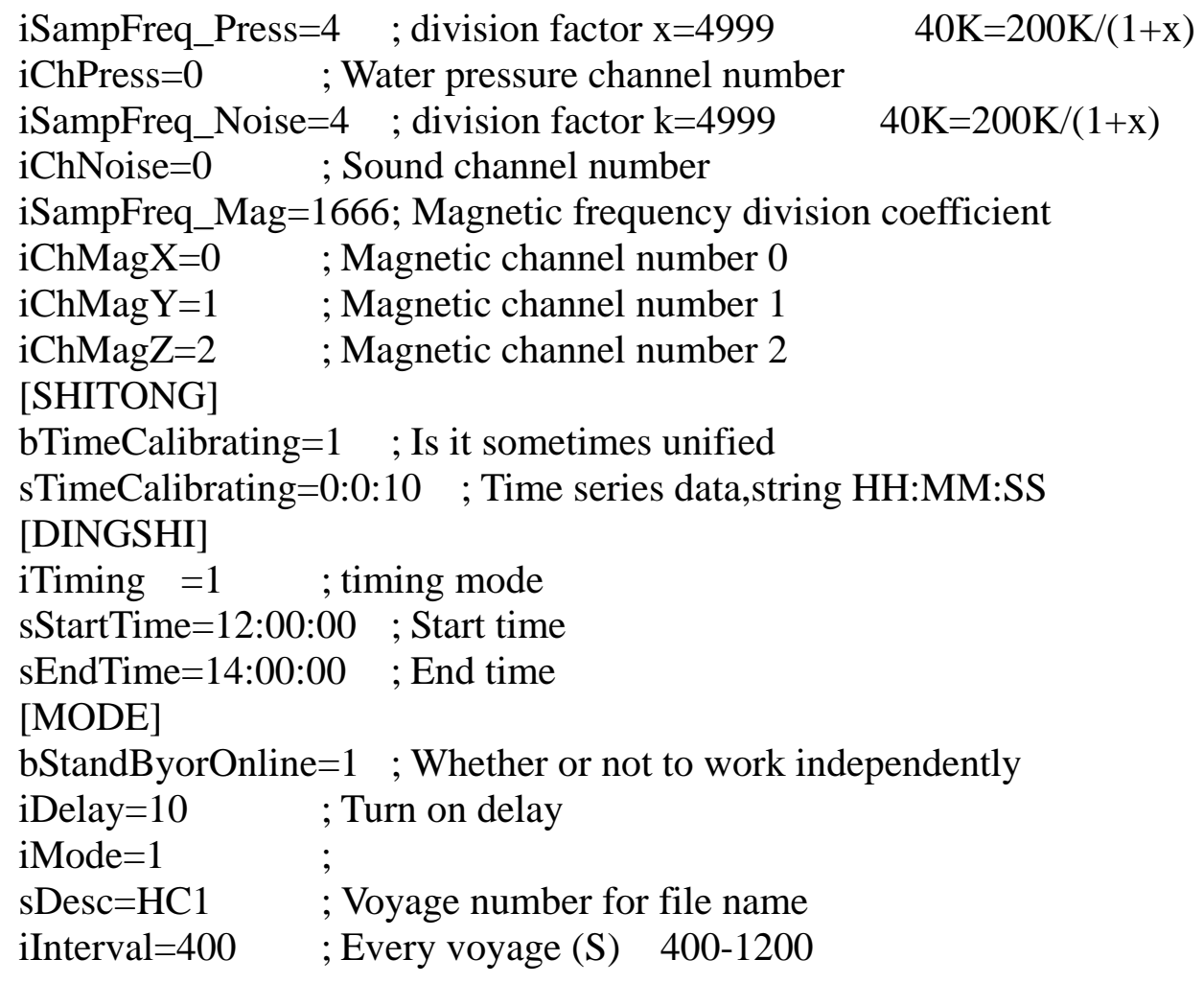

\section{Conclusion}

This paper presents a design of underwater physical field signal acquisition system, which can collect and preserve the acoustic signal, magnetic signal and water pressure signal produced in the underwater vehicle test. The requirements of the system are analyzed, the overall framework is given, and the implementation details of the system are introduced from the aspect of software structure and configuration file.

\section{References}

[1] Mcumber W E, CHENG B H. A General Framework for Formalizing UML with Formal Languages[C]. Proc. Int'l Conf Software Eng(ICSE2016), Michigan: IEEE, 2016:433-442.

[2] Campbell L A, CHENG B H, Mcumber W E, et al. Automatically Detecting and Visualizing Errors in UML Diagrams [J].Requirements Eng J(S0947-3602),2012,7(4):264-287.

[3] Konrad S, CHENG B H. Automated Analysis of Natural Language Properties for UML Models [J/OL]. http://www.cse.msu.edu/ konradsa, 2015-12-20.

[4] Hassan G. Designing Concurrent, Distributed, and Real-Time Applications with UML [M]. Addison-Wesley, 2012.

[5] Demartini C, Iosif R, Sisto R. A deadlock detection tool for concurrent Java programs [J]. Software-Practice and Experience, 2016, 29(7): 577-603.

[6] Bensalem S,Havelund K.Dynamic deadlock analysis of multi-threaded programs[C].In Haifa Verification Conference, 2015: 208-223.

[7] Chaki S, Clarke E, Ouaknine J, et al. Concurrent software verification with states, events, and deadlocks[J]. Formal Aspects of Computing, 2015, 17(4): 461-483. 